\title{
Is Gadolinium Contrast Necessary for Pituitary MRI in the Evaluation of Pediatric Short Stature and Growth Hormone Deficiency?
}

\author{
Abdullah Almaghraby ${ }^{a, b} \quad$ Alok Jajuc $^{c}$ Maura E. Ryan ${ }^{c}$ Karen Rychlik $^{b, d}$ \\ Reema L. Habiby ${ }^{a}$ b Wendy J. Brickman ${ }^{a, b}$ \\ ${ }^{a}$ Division of Endocrinology, Ann \& Robert H. Lurie Children's Hospital of Chicago, Chicago, IL, USA; bepartment of \\ Pediatrics, Northwestern University, Feinberg School of Medicine, Chicago, IL, USA; 'Division of Neuroradiology, \\ Ann \& Robert H. Lurie Children's Hospital of Chicago, Chicago, IL, USA; 'Stanley Manne Children's Research Institute, \\ Biostatistics Research Core, Ann \& Robert H. Lurie Children's Hospital of Chicago, Chicago, IL, USA
}

\section{Keywords}

Growth hormone deficiency - Magnetic resonance imaging • Short stature Short normal children

\begin{abstract}
Introduction: Short stature is a common concern that necessitates pediatric endocrinology evaluation. Growth hormone deficiency (GHD) is a commonly considered etiology. Brain and pituitary magnetic resonance imaging (MRI) with gadolinium-based contrast agents (GBCAs) is the most widely used imaging in assessing patients with GHD. Given the significant strides made in MRI technology, the need for contrast material should be reassessed. Method: We performed a retrospective review of healthy patients with short stature and/or GHD who underwent brain and pituitary MRI with and without contrast to assess the added value of contrast administration. Results: $227 / 318$ identified patients underwent growth hormone (GH) stimulation testing; 28 (12.3\%) with normal GH response and 62 (27.3\%) with severe GHD. We found a low incidence of sellar and suprasellar pathologies. When comparing noncontrast and contrast MRI, we found perfect agreement in detecting abnormal posterior pituitary bright spots (kappa:1.0) and substantial agreement
\end{abstract}

karger@karger.com

(c) 2021 S. Karger AG, Basel

www.karger.com/hrp

Karger" in detecting pars intermedia cysts and posterior superior sellar cysts (kappa: 0.74 and 0.71 , respectively). Initially, only moderate agreement was found in detecting infundibular abnormalities (kappa: 0.51), although a revised noncontrast MRI protocol with high-resolution 3D images enabled visualization of the infundibulum. Conclusion: The MRI evaluation of healthy patients with short stature and/or isolated GHD may be completed without the use of GBCAs. The slight overestimation of pituitary stalk interruption by noncontrast images can be overcome by adding newer high-resolution sequences.

ㄷ) 2021 S. Karger AG, Basel

\section{Introduction}

Short stature is a common concern that may require a pediatric endocrinology evaluation. The etiologies of short stature are diverse. Growth hormone deficiency accounts for $1-2 \%$ of short stature with a prevalence of approximately 1:3,500 [1]. The majority of GHD cases are idiopathic in nature, with only $20 \%$ due to organic causes, which include congenital central nervous system (CNS) anomalies, tumors, and other acquired pathological con- 
ditions that involve the pituitary-hypothalamic axis (HPA) $[2,3]$. The diagnosis of GHD involves laboratory evaluation, provocative testing, and neuroradiological assessment [4]. The radiological assessment of the HPA is considered the standard of care for evaluating patients with GHD [5]. Although brain and pituitary MRI is the imaging modality of choice in assessing these patients due to its noninvasiveness and its high spatial resolution, CNS pathologies are rarely found in such patients. In a study of 40 patients with isolated GHD, 35 (87.5\%) had normal brain MRIs [3]. The abnormal brain MRI findings include congenital anomalies such as pituitary hypoplasia (pituitary height $<-2$ standard deviation [SD] for age and sex), agenesis of the infundibulum, and absent or ectopic neurohypophysis [6,7].

Traditionally, these brain and pituitary MRI images are obtained with the use of gadolinium-based contrast agents (GBCAs). The main advantage of using contrast material is for the detection of small lesions, including pituitary microadenomas [8]. However, pituitary microadenomas generally do not cause GHD, and their presence is not considered a contraindication to treatment with growth hormone (GH) [9]. Furthermore, it has been shown that primary brain tumors are easily detected on noncontrast brain MRI images without the need to use GBCAs [9].

Given the concerns for potential health risks associated with GBCAs, the fact that pituitary microadenomas do not appear to be associated with GHD, and the ability of noncontrast brain MRI images in detecting primary brain tumors, the necessity of using contrast material when obtaining brain and pituitary MRI in cases of short stature and/or isolated GHD should be reassessed. There are 2 forms of GBCAs used in routine clinical practice: linear (such as gadopentetate dimeglumine) and macrocyclic (such as gadoterate meglumine). Nephrogenic systemic fibrosis, first reported in 2006, is a rare but serious condition linked to the administration of linear GBCAs in patients with renal failure [10]. The risk of developing this syndrome has been markedly reduced with the use of new macrocyclic GBCAs and routine screening of renal function prior to contrast administration [11]. More recently, deposition of GBCAs has been found in different tissues, including the brain, independent of renal function. The risk increases with repeated doses and with the use of linear agents, although deposition has been demonstrated with macrocyclics as well [11]. The clinical significance of this deposition is unclear but warrants caution, especially in children, who have a longer expected lifespan to manifest delayed effects. Allergic reactions and gastrointestinal symptoms in pediatric patients can occur with GBCA administration, although the incidence is low [12]. The use of contrast material also increases the total cost of the MRI study (USD 2,350 vs. USD 3,900 at our institution) and prolongs the time of the examination by about 15-20 min, which is of further concern if the child requires general anesthesia. Moreover, administration of contrast material requires intravenous access, causing discomfort and additional stress to children and their families.

We performed a retrospective review of otherwise healthy patients with short stature and/or isolated GHD who underwent brain and pituitary MRI with and without contrast at our institution from January 2013 to December 2018. The main goal was to assess whether contrast administration led to diagnoses that would have otherwise been missed and/or impacted the patient's clinical course.

\section{Hypothesis}

The use of GBCAs when obtaining brain and pituitary MRI does not increase the radiological detection of clinically significant HPA abnormalities in children with short stature and/or isolated GHD.

\section{Materials and Methods}

The study was approved by Ann \& Robert H. Lurie Children's Hospital of the Chicago Institutional Review Board. The requirement for informed consent was waived for this retrospective study.

\section{Patient Recruitment}

We queried the hospital's electronic medical record $\left(\mathrm{EPIC}^{\circledR}\right)$ by using the following diagnoses: short stature, GHD, and idiopathic short stature for patients who underwent brain and pituitary MRI with and without contrast from January 2013 to December 2018. We collected the following information from the charts: patient's current age, sex, patient's age and height at the time of MRI study, IGF-1 level at the time of MRI study, peak GH level after undergoing 2 provocative $\mathrm{GH}$ tests (carried out by administering insulin regular human 1:10 [humuLIN R], and Arginine R [GENE]), and other associated diagnoses. We included patients who have short stature defined as a height percentile $<3$ rd or have GHD defined as an IGF-1 $z$-score $<-2$ SD for age and/or GH provocative testing with a peak $\mathrm{GH}$ level of $<10 \mathrm{ng} / \mathrm{dL}$ [13]. We excluded patients with known genetic disorders, neurological disorders, tumors, or malignancies of any type, or patients with multiple pituitary hormone deficiencies known prior to obtaining the MRI study from our cohort [14]. The GHD cohort was then subgrouped into severe GHD ( $\mathrm{GH}$ peak $<5 \mathrm{ng} / \mathrm{mL}$ ), moderate GHD ( $\mathrm{GH}$ peak 5-7.99 ng/mL), and mild GHD (8-9.99 ng/mL) [15-17]. 


\section{MRI Evaluation}

Comparison of Pre-Contrast and Post-Contrast Imaging

MRI was performed on either $3 \mathrm{~T}$ or $1.5 \mathrm{~T}$ scanners (3T Skyra or 1.5T Aera, Siemens Healthineers, Erlangen, Germany or Signa Explorer 1.5 T, GE Healthcare, Chicago, IL, USA). Dedicated imaging through the pituitary gland included 3-mm-slice thickness, small field-of-view sagittal and coronal pre-contrast T1-weighted fat-saturated, dynamic post-contrast sagittal T1-weighted (4 runs), delayed post-contrast sagittal T1-weighted, and sagittal and coronal T2-weighted images. Images of the whole brain included precontrast sagittal T1-weighted, axial T2-weighted, axial fluid-attenuated inversion recovery, axial diffusion-weighted with apparent diffusion coefficient maps, axial gradient echo or susceptibilityweighted, and post-contrast axial T1-weighted or sagittal 3D T1weighted images with reconstructions.

The MRI studies were reviewed by 2 pediatric neuroradiologists. Each one independently reviewed half of the cohort's brain and pituitary MRI studies blinded to the clinical laboratory data. Each radiologist initially reviewed only the noncontrast portions of the study (Read A), and subsequently, the same radiologist reviewed the entire study, including pre- and post-contrast portions in a separate session (Read B). The 2 sessions occurred 6 weeks apart to avoid recall bias. To assess the inter-rater reliability, 60 studies were read by both radiologists.

The neuroradiologists systematically analyzed the following findings:

- Pituitary gland: height of the gland, presence and location of pre-contrast T1 bright spot, and the presence of any focal lesions (cyst or mass)

- Infundibulum: thickness, location, and enhancement

- Suprasellar region: appearance of optic chiasm (normal or hypoplastic), presence of any mass, if present, location, size, and enhancement

- Brain: septum pellucidum (present or absent), corpus callosum (present or absent and normal or dysgenesis), and any incidental abnormalities

\section{Modified Noncontrast High-Resolution Protocol}

MRI studies on an additional 15 patients with GHD were imaged with a modified noncontrast MRI protocol including highresolution 3D imaging (constructive interference steady state [CISS]) and were reviewed by both pediatric neuroradiologists separately. A modified noncontrast MRI protocol was implemented in our department in 2018 and is assessed separately in this study. This protocol included a high-resolution sagittal 3D T2weighted gradient echo sequence through the pituitary gland (CISS or fast imaging employing steady-state acquisition [FIESTA]), as well as the routine $3-\mathrm{mm}$-slice thickness and small field of view sagittal and coronal T1- and T2-weighted images. In addition to the pre-contrast brain sequences mentioned before, sagittal 3D T1 gradient-echo (magnetization prepared rapid gradient echo [MPRAGE] or brain volume imaging [BRAVO]) images through the entire brain were also obtained with axial and coronal reconstructions. MRI studies on an additional 15 patients with GHD were imaged with this high-resolution protocol. Images were reviewed by both pediatric neuroradiologists separately.

Statistical Analysis

The pituitary height $\mathrm{z}$-score for each patient was calculated based on the following equation: $z$-score $=($ raw score - population
Table 1. Patients' characteristics

\begin{tabular}{ll}
\hline Patients' characteristic & Mean, SD, and range \\
\hline Age at imaging, years $(N=318)$ & $10 \pm 3.4(1.1-18)$ \\
Height $z$-score $(N=318)$ & $-2.2 \pm 0.7(-4$ to -0.1$)$ \\
IGF-1 $z$-score $(N=316)$ & $-1 \pm 1.1(-3$ to 2.95$)$ \\
GH peak, ng $/ \mathrm{mL}(N=227)$ & $6.99 \pm 3.23(0.25-20.8)$ \\
Pituitary height, mm $(N=318)$ & $4.2 \pm 1.3(1-10)$ \\
Pituitary height $z$-score $(N=318)$ & $-0.5 \pm 1.3(-4.1-4.4)$ \\
\hline Patients' characteristics & $N(\%)$ \\
\hline Sex (female) & $101(32)$ \\
Stature $<3 r d$ percentile $(N=318)$ & $230(72)$ \\
IGF-1 $<-2$ SD $(N=316)$ & $76(24)$ \\
GH deficiency (mild, moderate, and & $23,37,27$ \\
$\quad$ severe; $N=227)$ & \\
\hline
\end{tabular}

$\mathrm{GH}$, growth hormone; SD, standard deviation.

mean)/standard deviation for age and sex based on the normative data identified by Sari et al. [18]. We performed descriptive statistics including frequencies and percentages for categorical data and means and SDs for continuous data, which were all normally distributed. $\chi^{2}$ statistics were used to compare the prevalence of MR findings in those with and without severe GH deficiency. To determine agreement between images read with contrast and the ones without, simple kappa coefficients were calculated for agreement for pre and post MRI images using the following reference ranges [19].

\section{Kappa Agreement}

- $<0.4$ less than chance agreement

- 0.41-0.6 moderate agreement

- $0.61-0.8$ substantial agreement $0.81-1.0$ almost perfect or perfect agreement

Intraclass correlation coefficients were run to determine interrater reliability for the number of congenital CNS cysts and abnormal infundibula by 2 different raters. All statistical tests assumed a 2 -sided tail, and $p$ values $<0.05$ were considered statistically significant. Analyses were performed using SAS version 9.4 (SAS Institute Inc, Cary, NC, USA).

\section{Results}

Of the 1,277 patients initially queried, 800 were excluded due to duplicate charts, 100 due to no MRI images available or not done, and 59 with known brain abnormalities or multiple pituitary hormone deficiencies. We identified 318 patients who met the inclusion criteria. One hundred one (32\%) patients were female. The mean age at the time of obtaining MRI was $10 \pm 3.4$ years (Table 1). Two hundred thirty (72.3\%) patients were 
Table 2. Frequency of MRI findings based on GH status

\begin{tabular}{|c|c|c|c|c|}
\hline \multirow[t]{2}{*}{ Finding } & \multicolumn{2}{|c|}{ MRI findings in normal $\mathrm{GH}(N=28)$} & \multicolumn{2}{|c|}{ MRI findings in GHD ( $N=199)$} \\
\hline & read $A, n(\%)$ & read $B, n(\%)$ & read $A, n(\%)$ & read $\mathrm{B}, n(\%)$ \\
\hline Pars intermedia cyst & $1(3.6)$ & $1(3.6)$ & $32(16.1)$ & $23(11.6)$ \\
\hline Chiari 1 malformation & $2(7.1)$ & $2(7.1)$ & $12(6.0)$ & $12(6.0)$ \\
\hline Abnormal infundibulum & $2(7.1)$ & $1(3.6)$ & $19(9.5)$ & $7(3.5)$ \\
\hline Pineal gland cyst & $1(3.6)$ & $1(3.6)$ & $5(2.5)$ & $5(2.5)$ \\
\hline Posterior superior cyst & $4(14.3)$ & $2(7.1)$ & $14(7.0)$ & $10(5.0)$ \\
\hline Gliosis & $1(3.6)$ & $1(3.6)$ & $7(3.5)$ & $7(3.5)$ \\
\hline Ectopic bright spot & 0 & 0 & $2(1.0)$ & $2(1.0)$ \\
\hline Arachnoid cyst & 0 & 0 & $4(2.0)$ & $4(2.0)$ \\
\hline Temporal lobe cyst & 0 & 0 & $2(1.0)$ & $2(1.0)$ \\
\hline Thalamic cyst & 0 & 0 & $3(1.5)$ & $3(1.5)$ \\
\hline Absent bright spot & 0 & 0 & 0 & 0 \\
\hline Ventriculomegaly & $1(3.6)$ & $1(3.6)$ & $1(0.5)$ & $1(0.5)$ \\
\hline Thin optic nerve & 0 & 0 & 0 & 0 \\
\hline
\end{tabular}

$\mathrm{GH}$, growth hormone; MRI, magnetic resonance imaging.

Table 3. Frequency of MRI findings based on the severity of GHD

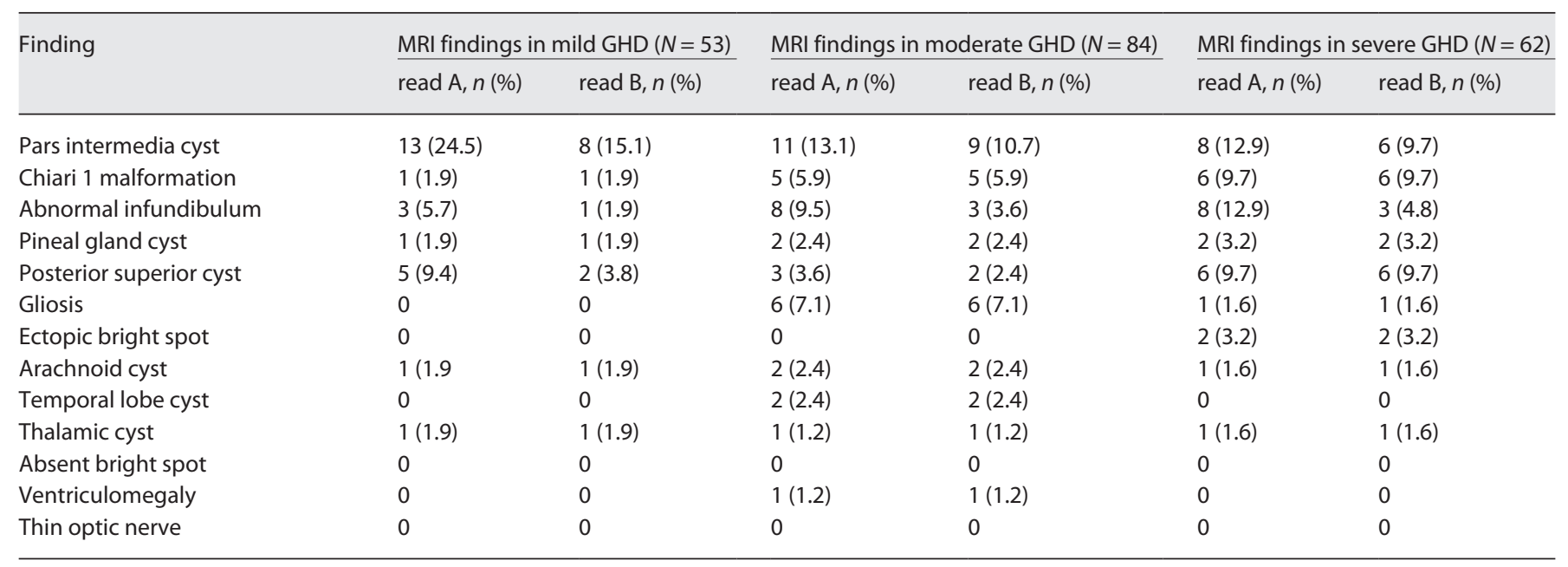

GHD, growth hormone deficiency; MRI, magnetic resonance imaging.

short in stature, with a height percentile $<3 \mathrm{rd}$. The mean height $z$-score in our population was $-2.2 \pm 0.7$. Three hundred sixteen (99.4\%) patients had IGF-1 testing done. Of those tested, 76 (24.1\%) had a $z$-score for age $<-2$ SD. The mean IGF-1 $z$-score for age in our cohort was $-1 \pm$ 1.1. Two hundred twenty-seven (71.4\%) patients underwent GH provocative testing, and each had 2 tests performed, as described in the method section. The mean peak $\mathrm{GH}$ was $6.99 \mathrm{ng} / \mathrm{mL} \pm 3.23$. Among patients who underwent $\mathrm{GH}$ provocative testing, 28 (12.3\%) patients had normal GH response and $62(27.3 \%)$ patients had severe GHD.

The MRI findings observed in our entire study population based on GH status are summarized in Table 2. We further broke down the findings based on the severity of GHD in Table 3. The pituitary findings included pars intermedia cysts between the anterior and posterior pituitary tissue (shown in Fig. 1), cysts along the posterior superior pituitary gland (shown in Fig. 2), ectopic posterior pituitary bright spot, and poorly visualized infundib- 
Fig. 1. T2 (a) and post-contrast T1 (b) images demonstrating a small nonenhancing focus between the anterior and posterior pituitary tissue indicative of a proteinaceous pars intermedia cyst.

Fig. 2. T2 (a) and post contrast T1 (b) images in a study patient with a small cyst along the posterior superior aspect of the pituitary gland.

Fig. 3. Four-year-old boy with short stature. Pre-contrast (a) and post-contrast (b) images demonstrate ectopic posterior pituitary tissue along the hypothalamus (arrows). There is no intrasellar posterior pituitary bright spot present. The infundibulum is not visible.
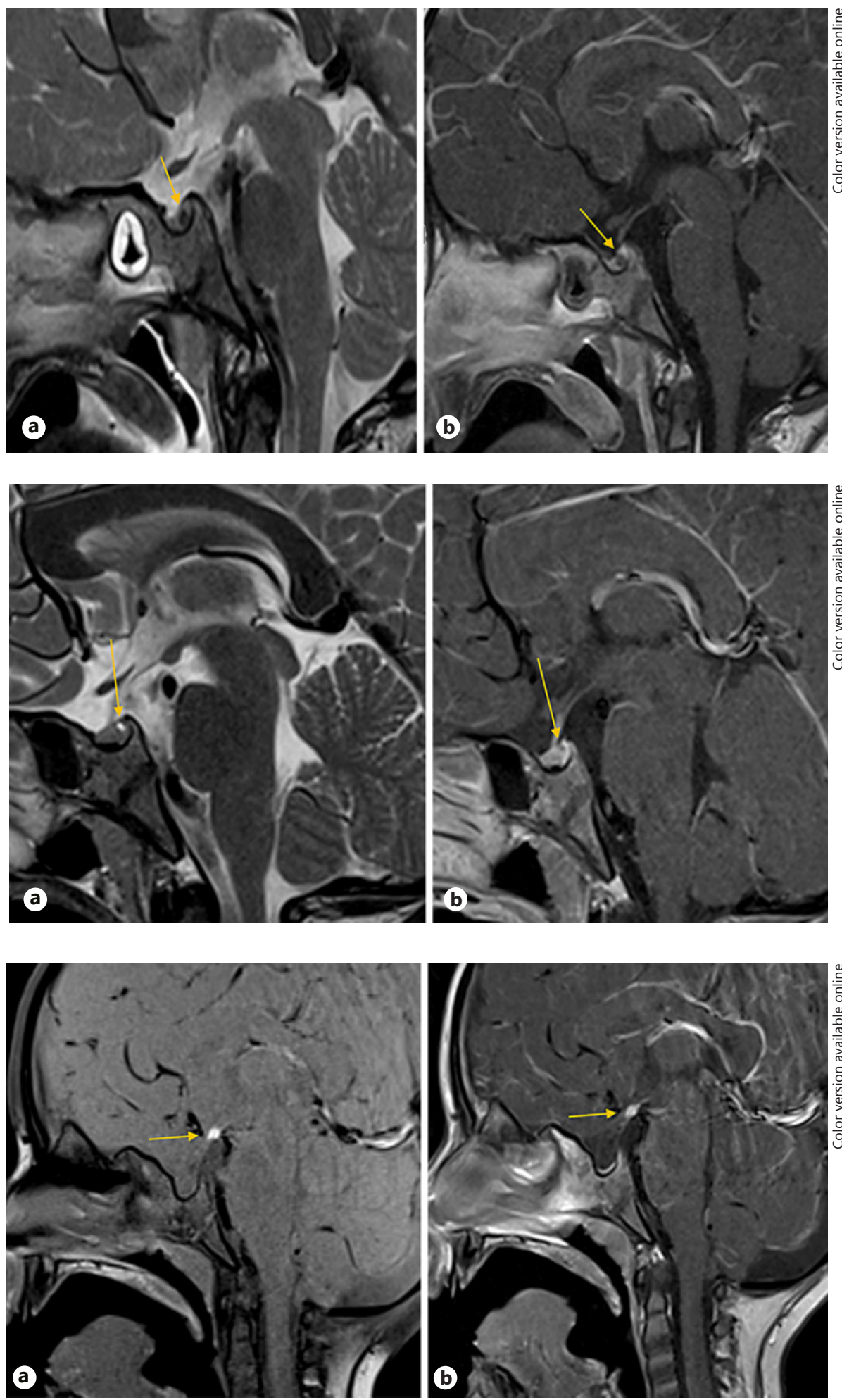

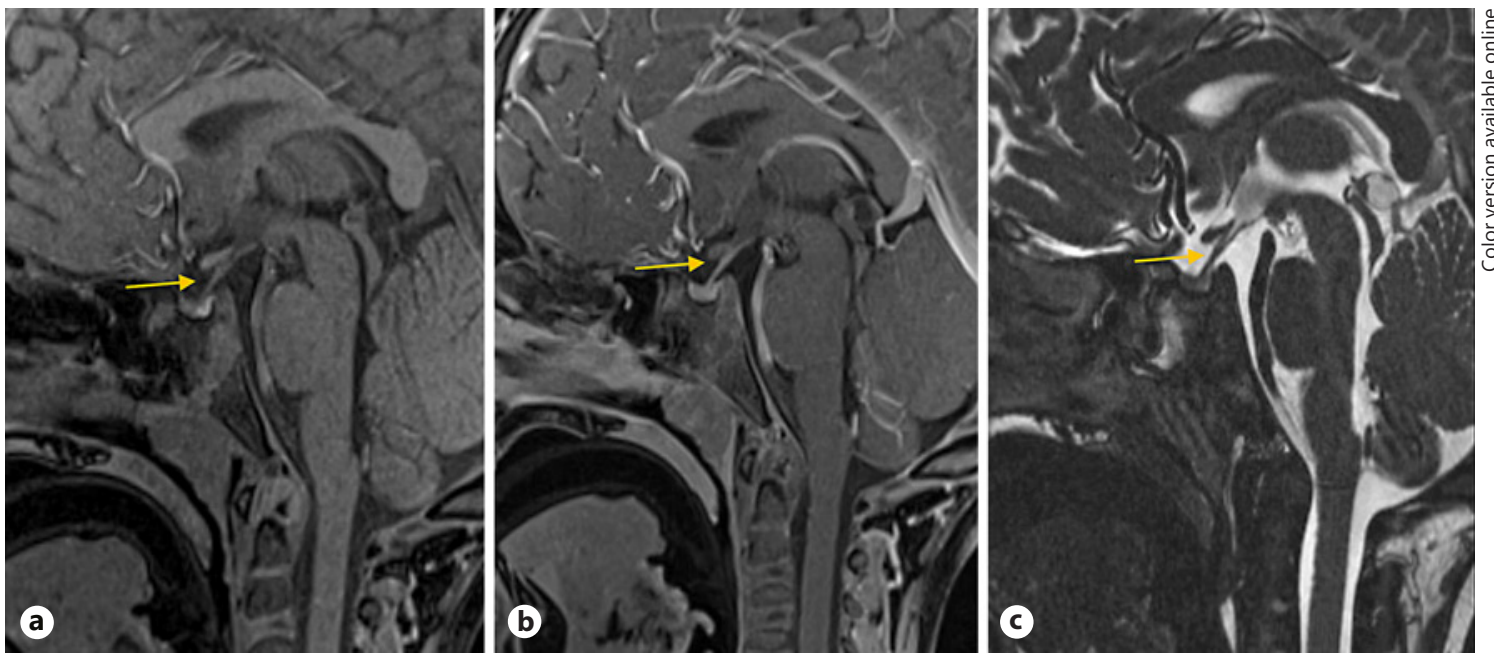

Fig. 4. This is an example of a normal stalk on pre-contrast T1 (a), post contrast T1 (b) and 3D FIESTA (c) images.

Table 4. Impact of using GBCAs on MRI findings in all subjects and in subjects with severe GHD

\begin{tabular}{lll}
\hline Finding & $\begin{array}{l}\text { Kappa }(95 \% \mathrm{Cl}){ }^{*} \\
\text { all subjects }\end{array}$ & $\begin{array}{l}\text { Kappa }(95 \% \mathrm{Cl}) * \\
\text { in severe GHD subjects }\end{array}$ \\
\hline Pars intermedia cyst & $0.74(0.63-0.85)$ & $0.77(0.53-1.0)$ \\
Posterior superior cyst & $0.71(0.54-0.89)$ & $1.0(1.0-1.0)$ \\
Pineal gland cyst & $1.0(1.0-1.0)$ & $1.0(1.0-1.0)$ \\
Arachnoid cyst & $0.91(0.73-1.0)$ & $1.0(1.0-1.0)$ \\
Temporal lobe cyst & $1.0(1.0-1.0)$ & $1.0(1.0-1.0)$ \\
Thalamic cyst & $1.0(1.0-1.0)$ & $0.51(0.15-0.87)$ \\
Abnormal infundibulum (absent, thin, and thick) & $0.51(0.34-0.67)$ & $1.0(1.0-1.0)$ \\
Abnormal posterior bright spot (absent/ectopic) & $1.0(1.0-1.0)$ & \\
\hline
\end{tabular}

* $p<0.0001$. GHD, growth hormone deficiency; MRI, magnetic resonance imaging; $\mathrm{Cl}$, confidence interval; GBCAs, gadolinium-based contrast agents.

ula (shown in Fig. 3). It is worth mentioning that there were no intrasellar lesions or adenomas identified. The mean pituitary height was $4.2 \mathrm{~mm} \pm 1.3$, with a mean $z$ score of $-0.5 \pm 1.3$ [18]. Of note, the frequency of abnormalities, as well as the frequency of failing to visualize the infundibulum, was similar on $3 \mathrm{~T}$ and $1.5 \mathrm{~T}$ (data not shown).

The levels of agreement between the MRI interpretations based on noncontrast images compared to with and without contrast images are summarized in Table 4 . We found perfect agreement in detecting an abnormal posterior pituitary bright spot and substantial agreement in detecting pars intermedia cysts and posterior superior sellar cysts. Only moderate agreement was found in detecting infundibular abnormalities. For the detection of incidental intracranial cysts including pineal gland cysts, arachnoid cysts, temporal lobe cysts, and thalamic cysts, there was a near perfect agreement.

In the subsequent group of GHD patients imaged with the modified noncontrast high-resolution proto$\mathrm{col}$, the infundibulum was visualized in $13 / 15$ patients $(87 \%)$ on $3 \mathrm{D}$ CISS imaging yet could be seen in the remaining 2 on corresponding 3D T1 images (shown in Fig. 4). In one child, the stalk was obscured on CISS imaging by orthodontic artifact. In another child, the stalk was difficult to distinguish from the adjacent anterior clinoid bone on CISS sequences (shown in Fig. 5). We evaluated the level of agreement (inter-rater reli- 
Fig. 5. A thirteen-year-old boy with short stature. On 3D CISS imaging (a), the infundibulum is closely opposed to the clinoid bone and difficult to distinguish. The stalk is visible on the corresponding sagittal $3 \mathrm{D}$ $\mathrm{T} 1$ and coronal reformatted images $(\mathbf{b}, \mathbf{c})$.
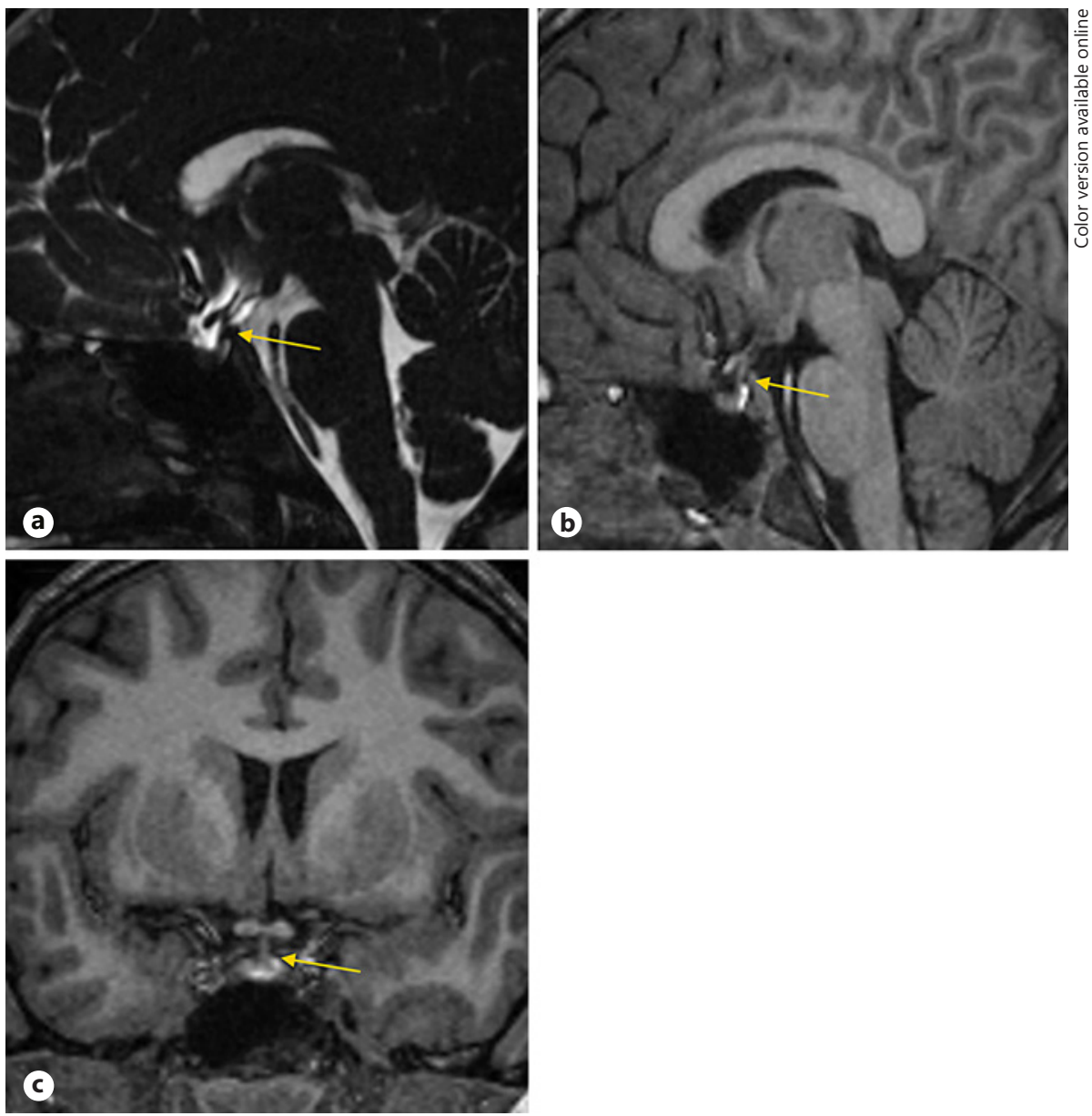

ability) between the 2 neuroradiologists and found almost perfect agreement (kappa: 0.902, $p$ value: $<0.0001$ ) between their reads.

\section{Discussion/Conclusion}

To our knowledge, this is the first study to examine the utility of MRI contrast material in the evaluation of pediatric short stature and/or isolated GHD. Our study identified a low incidence of pituitary and suprasellar abnormalities in these children, which is consistent with findings from prior studies $[3,20]$. The abnormalities found in our study are mainly developmental anomalies of the gland and congenital cysts, with no pituitary adenomas or suprasellar masses identified. We found near-perfect to perfect agreement in identification of these abnormalities on noncontrast MRI compared to with and without contrast MRI, except for visualization of the infundibulum, for which we found moderate agreement.
The noncontrast images seemed to overestimate the infundibular abnormalities, with abnormal infundibula in $6 \%$ of our cohort based on noncontrast MRI and $4.7 \%$ based on with and without contrast MRI. Accurate assessment of the infundibulum can have important clinical significance as these abnormalities have been associated with multiple pituitary hormone dysfunctions and may prompt more frequent screening of hypothalamic/pituitary function [21]. The use of contrast appeared to improve the visualization of the infundibula compared to noncontrast sequences on the routine $3-\mathrm{mm}$-slice thickness pituitary images. However, this limitation of noncontrast imaging can be mitigated by the use of newer high-resolution volumetric sequences. Examples of 3D T2-weighted sequences depending on the MRI vendor are CISS, FIESTA, and driven equilibrium, which can provide sub-millimeter slice thickness and exquisite anatomic detail without the need for GBCAs [22]. Additionally, 3D T1 images (like MPRAGE or BRAVO) which are now routine part of most brain MRI imaging protocols can also provide high spatial 
resolution needed for delineation of the pituitary infundibulum. More recently, our institution has added midline, sagittal plane, high-resolution T2-weighted CISS/FIESTA images and whole-brain 3D T1 MPRAGE/BRAVO images in all pituitary MRI protocols. In our review of 15 patients who underwent imaging as part of this new protocol, we were able to confidently visualize the infundibulum in all cases, with the 3D T2-weighted and 3D T1weighted images being complementary to each other. This suggests that imaging assessment of the infundibulum is not necessarily dependent on contrast enhancement but rather on the spatial resolution of MRI, and we believe that noncontrast high-resolution images can visualize the infundibulum and any associated abnormalities. One pitfall of CISS/FIESTA imaging is the greater susceptibility to metal-related artifact, which can be an issue in children wearing dental braces.

Abnormalities of the posterior bright spot such as absent, ectopic, or split, which can cause GHD by interrupting the HPA, were identified reliably by both noncontrast and with contrast images. These abnormalities were seen in $2.5 \%$ of our cohort, which is slightly higher than previously published prevalence [23].

The most frequent sellar finding identified in our study was the presence of small cysts (14.8\%), either at the junction of the anterior and posterior pituitary $(11 \%)$ or along the posterior superior aspect of the sella (3.8\%). The cysts at the junction of the anterior and posterior pituitary are typically described as pars intermedia/Rathke's cleft cysts, thought to arise from the ectoderm, and have been reported in up to $57 \%$ of children using modern high-resolution MRI [24]. Autopsy studies have reported the prevalence of Rathke's cleft cysts in $12-33 \%$ of adults [25]. These cysts are thought to be incidental findings and rarely of any clinical significance [26]. Our study also did not find any significant relationship between the presence of these cysts and severe GH deficiency.

We did not find any specific descriptions of the "posterior-superior" sellar cysts in the published literature. However, based on the findings in this study and empirical experience of the readers, we hypothesize that these are relatively common developmental cysts, possibly arising from the diencephalon, without clinical significance. The high concordance between the noncontrast and contrast images in identification of both of these cyst types on our study suggests that the use of GBCAs does not have any added benefits in evaluating congenital pituitary cysts.

The use of brain and pituitary MRI with and without contrast is still the recommended imaging modality based on the recent consensus guidelines for the diagnosis and treatment of pediatric GHD [27]. Given the increasing recognition of GBCA deposition in different body tissues and the associated potential health risks [28-31], the added financial burden from contrast administration and the longer scan times, particularly for children under general anesthesia, the need to use contrast material when imaging healthy children with short stature and/or GH deficiency should be reconsidered. Our study provides valuable data that allow the clinicians to make informed decisions regarding the choice of imaging.

The limitations of our study include the following: there were only 2 readers for each of the $318 \mathrm{MRI}$ images; however, agreement between the raters for the 60 images read by both radiologists was significant, though lower than ideal. In addition, we did not have any subjects with both traditional pre- and post-contrast images as well as the modified high-resolution images.

In conclusion, the neuroradiological evaluation of healthy patients with short stature and/or isolated GHD can be done by MRI without the use of GBCAs, thus avoiding the potential risks and added costs. The incidence of pathologies identified by MRI in these patients is low, with the majority of structural abnormalities adequately identified by noncontrast sequences. Routine noncontrast MRI sequences may lead to overdiagnosis of pituitary stalk interruption; however, this can be circumvented by the inclusion of high-resolution, volumetric T2 sequence, using CISS, FIESTA, or driven equilibrium as well as MPRAGE or BRAVO, components of which are now standard practice at our institution. Sub-centimeter pituitary cysts are commonly seen at the junction of anterior and posterior lobes or along the posterior superior margin of the gland, both of which are likely not clinically significant. Although no suprasellar masses were identified in our study, the identification of a mass may necessitate repeat imaging with contrast. Our findings suggest the risks of using GBCAs for assessment of short stature in GHD may outweigh the benefits.

\section{Statement of Ethics}

The study was approved by Ann \& Robert H. Lurie Children's Hospital of the Chicago Institutional Review Board (IRB: 20192593). The requirement for informed consent was waived for this retrospective study.

\section{Conflict of Interest Statement}

All the authors have no conflict of interest to disclose. 


\section{Funding Sources}

No funding was secured for this research project.

\section{Author Contributions}

Abdullah Almaghraby collected the clinical information for the cohort, conducted the literature review, drafted the initial manuscript, and edited the manuscript according to feedback of the other authors. Alok Jaju read the brain and pituitary MRIs for this study, along with Dr. Ryan. He provided feedback and reviewed and revised the manuscript. Maura E. Ryan read the brain and pituitary MRIs for this study, along with Dr. Jaju. She provided feed- back and reviewed and revised the manuscript. Karen Rychlik performed the statistical analyses for this project. Reema L. Habiby provided feedback and critically reviewed the manuscript. Wendy J. Brickman supervised and organized the whole project. She provided feedback about and reviewed and revised the manuscript. All the authors approved the final manuscript as submitted and agreed to be accountable for all aspects of the work.

\section{Data Availability Statement}

All data generated or analyzed during this study are included in this. Further inquiries can be directed to the corresponding author.

\section{References}

1 Lindsay R, Feldkamp M, Harris D, Robertson J, Rallison M. Utah growth study: growth standards and the prevalence of growth hormone deficiency. J Pediatr. 1994;125(1):2935.

2 Argente J. Challenges in the management of short stature. Horm Res Paediatr. 2016;85(1): 2-10.

3 Naderi F, Eslami SR, Mirak SA, Khak M, Amiri J, Beyrami B, et al. Effect of growth hormone deficiency on brain MRI findings among children with growth restrictions. J Pediatr Endocrinol Metab. 2015;28(1-2): $117-23$.

4 Stanley T. Diagnosis of growth hormone deficiency in childhood. Curr Opin Endocrinol Diabetes Obes. 2012;19(1):47-52.

5 Grimberg A, DiVall SA, Polychronakos C, Allen DB, Cohen LE, Quintos JB, et al. Guidelines for growth hormone and insulin-like growth factor-I treatment in children and adolescents: growth hormone deficiency, idiopathic short stature, and primary insulin-like growth factor-I deficiency. Horm Res Paediatr. 2016;86(6):361-97.

6 Bozzola M, Adamsbaum C, Biscaldi I, Zecca M, Cisternino M, Genovese E, et al. Role of magnetic resonance imaging in the diagnosis and prognosis of growth hormone deficiency. Clin Endocrinol. 1996;45(1):21-6.

7 Bozzola M, Mengarda F, Sartirana P, Tatò L, Chaussain JL. Long-term follow-up evaluation of magnetic resonance imaging in the prognosis of permanent GH deficiency. Eur J Endocrinol. 2000;143(4):493-6.

8 Chaudhary V, Bano S. Imaging of the pituitary: recent advances. Indian J Endocrinol Metab. 2011;15(3):S216-23.

9 Falk Delgado A, Van Westen D, Nilsson M, Knutsson L, Sundgren PC, Larsson EM, et al. Diagnostic value of alternative techniques to gadolinium-based contrast agents in MR neuroimaging-a comprehensive overview. Insights Imaging. 2019;10(1):84.
10 Marckmann P, Skov L, Rossen K, Dupont A, Damholt MB, Heaf JG, et al. Nephrogenic systemic fibrosis: suspected causative role of gadodiamide used for contrast-enhanced magnetic resonance imaging. J Am Soc Nephrol. 2006;17(9):2359-62.

11 Murata N, Gonzalez-Cuyar LF, Murata K, Fligner C, Dills R, Hippe D, et al. Macrocyclic and other non-group 1 gadolinium contrast agents deposit low levels of gadolinium in brain and bone tissue: preliminary results from 9 patients with normal renal function. Invest Radiol. 2016;51(7):447-53.

12 Neeley C, Moritz M, Brown JJ, Zhou Y. Acute side effects of three commonly used gadolinium contrast agents in the paediatric population. Br J Radiol. 2016;89(1063):20160027.

13 Hanew K, Utsumi A. The role of endogenous GHRH in arginine-, insulin-, clonidine- and 1-dopa-induced GH release in normal subjects. Eur J Endocrinol. 2002;146(2):197-202.

14 Bhargava R, Hahn G, Hirsch W, Kim MJ, Mentzel HJ, Olsen OE, et al. Contrast-enhanced magnetic resonance imaging in pediatric patients: review and recommendations for current practice. Magn Reson Insights. 2013;6:95-111.

15 Wagner IV, Paetzold C, Gausche R, Vogel M, Koerner A, Thiery J, et al. Clinical evidencebased cutoff limits for $\mathrm{GH}$ stimulation tests in children with a backup of results with reference to mass spectrometry. Eur J Endocrinol. 2014;171(3):389-97.

16 Collett-Solberg PF, Ambler G, Backeljauw PF, Bidlingmaier M, Biller BMK, Boguszewski MCS, et al. Diagnosis, genetics, and therapy of short stature in children: a growth hormone research society international perspective. Horm Res Paediatr. 2019;92(1):1-14.

17 S2e-Leitlinie 174/002 Aktualisierte Leitlinie. Diagnostik des Wachstumshormonmangels im Kindes- und Jugendalter. Kurzfassung Stand. 2014.
18 Sari S, Sari E, Akgun V, Ozcan E, Ince S, Saldir $\mathrm{M}$, et al. Measures of pituitary gland and stalk: from neonate to adolescence. J Pediatr Endocrinol Metab. 2014;27(11-12):1071-6.

19 Landis JR, Koch GG. The measurement of observer agreement for categorical data. Biometrics. 1977;33(1):159-74.

20 Schmitt J, Thornton P, Shah AN, Rahman AKMF, Kubota E, Rizzuto P, et al. Brain MRIs may be of low value in most children diagnosed with isolated growth hormone deficiency. J Pediatr Endocrinol Metab. 2021; 34(3):333-40.

21 Hamilton BE, Salzman KL, Osborn AG. Anatomic and pathologic spectrum of pituitary infundibulum lesions. Am J Roentgenol. 2007;188(3):W223-32.

22 Godano E, Morana G, Di Iorgi N, Pistorio A, Allegri AEM, Napoli F, et al. Role of MRI T2DRIVE in the assessment of pituitary stalk abnormalities without gadolinium in pituitary diseases. Eur J Endocrinol. 2018;178(6):61322.

23 Kara Ö, Esen I, Tepe D, Gülleroğlu NB, Tayfun $M$. Relevance of pituitary gland magnetic resonance imaging results with clini$\mathrm{cal}$ and laboratory findings in growth hormone deficiency. Med Sci Monit. 2018;24: 9473-8.

24 Mahdi ES, Webb RL, Whitehead MT. Prevalence of pituitary cysts in children using modern magnetic resonance imaging techniques. Pediatr Radiol. 2019;49(13):1781-7.

25 Jung JE, Jin J, Jung MK, Kwon A, Chae HW, $\mathrm{Kim} \mathrm{DH}$, et al. Clinical manifestations of Rathke's cleft cysts and their natural progression during 2 years in children and adolescents. Ann Pediatr Endocrinol Metab. 2017; 22(3):164-9.

26 Teramoto A, Hirakawa K, Sanno N, Osamura Y. Incidental pituitary lesions in 1,000 unselected autopsy specimens. Radiology. 1994; 193(1):161-4. 
27 Growth Hormone Research Society. Consensus guidelines for the diagnosis and treatment of growth hormone (GH) deficiency in childhood and adolescence: summary statement of the GH research society. GH research society. J Clin Endocrinol Metab. 2000;85(11):3990-3.

28 Lord ML, Chettle DR, Gräfe JL, Noseworthy MD, McNeill FE. Observed Deposition of gadolinium in bone using a new noninvasive in vivo biomedical device: results of a small pilot feasibility study. Radiology. 2018;287(1):96-103.

29 Roberts DR, Lindhorst SM, Welsh CT, Maravilla KR, Herring MN, Braun KA, et al. High levels of gadolinium deposition in the skin of a patient with normal renal function. Invest Radiol. 2016;51(5):280-9.

30 Maximova N, Gregori M, Zennaro F, Sonzogni A, Simeone R, Zanon D. Hepatic gad- olinium deposition and reversibility after contrast agent-enhanced MR imaging of pediatric hematopoietic stem cell transplant recipients. Radiology. 2016;281(2): 418-26.

31 Guo BJ, Yang ZL, Zhang LJ. Gadolinium deposition in brain: current scientific evidence and future perspectives. Front Mol Neurosci. 2018;11:335. 